\title{
Icaritin acts synergistically with epirubicin to suppress bladder cancer growth through inhibition of autophagy
}

\author{
XIU-WU PAN ${ }^{1,2^{*}}$, LIN LI $^{1,2^{*}}$, YI HUANG $^{1}$, HAI HUANG ${ }^{1}$, DAN-FENG XU $^{3}$, YI GAO $^{1}$, LU CHEN $^{1}$, \\ JI-ZHONG REN $^{1}$, JIAN-WEI CAO ${ }^{4}$, YI HONG ${ }^{1}$ and XIN-GANG CUI ${ }^{1,2}$
}

${ }^{1}$ Department of Urinary Surgery of Changzheng Hospital, Second Military Medical University, Huangpu, Shanghai 200003;

${ }^{2}$ Department of Urinary Surgery of the Third Affiliated Hospital, Second Military Medical University, Jianding District, Shanghai 201805; ${ }^{3}$ The Urology Research Center of the Chinese People's Liberation Army, Changzheng Hospital, Second Military Medical University, Huangpu; ${ }^{4}$ Navy No. 411 Hospital of PLA, Hongkou, Shanghai 200003, P.R. China

Received July 7, 2015; Accepted August 19, 2015

DOI: $10.3892 /$ or.2015.4335

\begin{abstract}
Bladder cancer is one of the most commonly diagnosed urological malignancies. Acquired resistance to chemotherapy is a great barrier for achieving successful treatment of bladder cancer. In the present study, we investigated the effect and mechanisms of icaritin, a flavonol glycoside derived from genus Epimedium, against human bladder cancer cells. It was found that despite the low cytotoxicity in normal human HEK293 cells, icaritin significantly inhibited the proliferation and colony formation of BT5637 and T24 bladder cancer cells time- and dose-dependently compared to the DMSO vehicle control. Moreover, cell viability monitored through mitochondrial membrane potential was inhibited markedly after icaritin treatment. Further investigation indicated that icaritin may inhibit epirubicin (EPI)-induced autophagy, and acted synergistically with EPI to suppress the proliferation of BT5637 and T24 cells. These findings suggest that icaritin may prove to be a novel potent therapeutic agent for the treatment of bladder cancer.
\end{abstract}

\section{Introduction}

Bladder cancer, second to prostate cancer, is a commonly diagnosed cancer of the genito-urinary system. According to 2014 cancer statistics of the American Cancer Society (ACS),

Correspondence to: Professor Xin-Gang Cui or Dr Yi Hong, Department of Urinary Surgery of the Third Affiliated Hospital, Second Military Medical University, Jianding District, Shanghai 201805, P.R. China

E-mail: xingangcui@126.com

E-mail: zandh@163.com

${ }^{*}$ Contributed equally

Abbreviations: DMSO, dimethylsulfoxide; EPI, anthracycline epirubicin; MMP, mitochondrial membrane potential; TUR, transurethral resection

Key words: bladder cancer, BT5637 cells, T24 cells, icaritin, autophagy, epirubicin
141,610 new cases of bladder cancer and 30,350 cancer-related deaths were estimated in the USA in the genito-urinary system (1). The most common type of bladder cancer tissue is transitional cell carcinoma, which accounts for more than $90 \%$ of all bladder carcinoma cases (2). Additionally, $\sim 70 \%$ transitional cell carcinomas are superficial tumors. Transurethral resection (TUR) is the primary regimen for patients with superficial bladder tumors (stages $\mathrm{T}_{\mathrm{a}}, \mathrm{T}_{1}$ or $\mathrm{T}_{\mathrm{is}}$ ) (3). However, the recurrence rate within 5 years after TUR is as high as $50-70 \%$ (4), and $10-20 \%$ of them progressed to muscle-invasive disease (5). The anthracycline epirubicin (EPI) is the first line treatment routinely used in patients with bladder cancer after TUR (6). Although prophylactic EPI can effectively reduce the recurrence rate of bladder cancer after TUR, the acquired drug resistance still remains a serious issue in bladder cancer patients.

Icaritin (Fig. 1A) is a hydrolytic form of icariin which is one of the traditional Chinese herbals (7). Icaritin has been shown to have a wide range of pharmacological and biological activities, including stimulating neuronal differentiation via estrogen-dependent pathway $(8,9)$, promoting differentiation of embryonic stem cells into cardiomyocytes $(10,11)$, inhibiting osteoclast differentiation (12) and promoting apoptosis and suppressing proliferation of prostatic smooth muscle cells (13). In addition, numerous studies have demonstrated that icaritin effectively promoted apoptosis, inhibited cell proliferation and induced cell cycle arrest in breast, endometrial and prostate cancer, and renal cancer cells (14-18). Icaritin is expected to work as an effective antitumor agent to regulate cancer cell growth via various pathways. However, the function of icaritin against bladder cancer has not been studied yet.

Autophagy has dual roles in cancer, acting as a tumor suppressor and as a mechanism of cell survival that can promote the growth of established tumors (19). In normal cells, a well regulated autophagy may prevent the accumulation of damaged proteins and organelles to protect cells from apoptosis or tumorigenesis. However, in tumor cells, autophagy will convey a pro-survival signal to adapt tumor cells to various adverse conditions, such as starvation and chemotoxicity. Autophagy is upregulated upon interferon- $\alpha$ (IFN- $\alpha$ ) treatment to induce IFN- $\alpha$ resistance in bladder cancer cells (20). In addition, 
autophagy protects breast cancer cells from EPI-induced apoptosis and facilitates epirubicin-resistance development (21). The p53-induced glycolysis and apoptosis regulator (TIGAR) inhibits autophagy, resulting in reduced sensitivity of cancer cells to epirubicin treatment (22). Several studies have reported that apoptosis of cancer cells is markedly enhanced by pharmacological or genetic mediated inhibition of autophagy (23-25). Furthermore, autophagy inhibitors such as 3-methyladenine or inhibition of autophagy regulatory pathways have been proved to sensitize cancer cells to chemotherapeutic agents (20-22).

The primary objective of the present investigation was to explore the antitumor molecular mechanism of icaritin in the treatment of human bladder cancer cells. To better understand the function of icaritin in treating bladder cancer, we studied the effects of icaritin on proliferation, apoptosis and autophagy of human bladder cancer BT5637 and T24 cells, and explored the sensitivity of cancer cells to EPI treatment.

\section{Materials and methods}

Reagents and antibodies. Icaritin (purity 98\%; Shanghai Yuanye Biotechnology Co., Ltd., China) was dissolved in dimethylsulfoxide (DMSO) solution to reach a concentration of $50 \mathrm{mM}$, and then stored at $-20^{\circ} \mathrm{C}$ before use. The stock solution was diluted to the final concentration using correspondent cell culture medium, and the final DMSO concentration in experimental culture medium was $<0.1 \%$. Therefore, $0.1 \%$ DMSO was used as control vehicle throughout the present study. Epirubicin was purchased from AdooQ BioScience (10 mg). The antibodies of ATG3 (\#3415), ATG5 (\#8540), ATG7 (\#2631) and LC3B (\#3868) were purchased from Cell Signaling Technology. GAPDH was purchased from ProteinTech Group (10494-1-AP).

Cell culture. The bladder cancer cell line BT5637 and T24 and the normal renal cell line HEK293 were purchased from the Cell Bank of Chinese Academy of Science. BT5637 cells were cultured in RPMI-1640 culture medium (Hyclone) with $10 \%$ fetal bovine serum (FBS), $1 \%$ non-essential amino acid (100X) and $2 \mathrm{~g} / 1$ glucose. T24 cells were cultured in McCoys' 5A culture medium (Sigma) with 10\% FBS. HEK293 cells were cultured in DMEM culture medium (HyClone) with 10\% FBS and $2 \mathrm{mmol} / \mathrm{l}$ glutamine. All these cells were cultured in a humidified atmosphere of $95 \%$ air and $5 \% \mathrm{CO}_{2}$ at $37^{\circ} \mathrm{C}$.

Cell proliferation assay. The 3-(4,5-dimethylthiazol-2-yl)2,5-diphenyltetrazolium bromide (MTT) assay was carried out to evaluate the viability and proliferation of cells. A total of $6 \times 10^{3}$ cells/well for BT5637 and HEK293 cells and $4 \times 10^{3}$ cells/well for T24 cells were, respectively, seeded into a 96-well plate, and incubated in respective culture medium for $24 \mathrm{~h}$ at $37^{\circ} \mathrm{C}$. Then culture medium was replaced with fresh culture medium containing varying concentrations of icaritin, and these cells were incubated for 24, 48 and $72 \mathrm{~h}$. At each time point, cells of each well were added with $20 \mu \mathrm{l}$ of $5 \mathrm{mg} / \mathrm{ml} \mathrm{MTT} \mathrm{solution} \mathrm{(Sigma).} \mathrm{After} 6$-h incubation at $37^{\circ} \mathrm{C}$, the formazan crystals were dissolved by adding $100 \mu \mathrm{l}$ DMSO into each well. The absorbance rate was measured at $570 \mathrm{~nm}$ using a spectrophotometer (Epoch Synergy 2; BioTek, USA). Each experiment was carried out in triplicates.
Colony formation assay. BT5637 and T24 cells were plated into 6-well plates $\left(6 \times 10^{2}\right.$ cells/well) in triplicate and were incubated for $24 \mathrm{~h}$ at $37^{\circ} \mathrm{C}$. The culture medium was then removed and fresh culture medium containing varying concentrations of icaritin was added into each well. After 48-h incubation at $37^{\circ} \mathrm{C}$, the special culture medium was changed to culture medium without icaritin. Cell culture was terminated when most colonies contained $>50$ cells. Subsequently, cells were washed twice with phosphate-buffered saline (PBS), fixed in $1 \mathrm{ml}$ paraformaldehyde for $30 \mathrm{~min}$, stained with $500 \mu \mathrm{l}$ Giemsa dye for $20 \mathrm{~min}$ and washed again with $\mathrm{ddH}_{2} \mathrm{O}$. Individual colonies were photographed using a microscope, the plates were photographed using a digital camera, and the colonies with $>50$ cells were counted. Each experiment was repeated three times.

Measurement of mitochondrial membrane potential (MMP). JC-1 (Beyotime) staining assay as previously described (26) was carried out to measure MMP. A total of $2 \times 10^{4}$ cells/well BT5637 or T24 cells were seeded into a $6 \mathrm{~cm}$ plate in triplicate and cultured at $37^{\circ} \mathrm{C}$. After $24-\mathrm{h}$ incubation, the culture medium was changed to fresh culture medium containing varying concentrations of icaritin and then cells were incubated for $72 \mathrm{~h}$ at $37^{\circ} \mathrm{C}$. At the designated time point, cells were trypsinized, resuspended, incubated with special culture medium containing JC-1 staining solution for $30 \mathrm{~min}$ at $37^{\circ} \mathrm{C}$, stained, washed twice using staining buffer and resuspended with $500 \mu \mathrm{l}$ staining buffer. Healthy cells with high-MMP in the form of JC-1 aggregates showed red fluorescence, and apoptotic cells with low-MMP in the form of JC-1 monomer emitted green fluorescence only. Finally, the stained cells were analyzed using flow cytometry (BD Biosciences).

Western blotting. The T24 and BT5637 cells were previously treated with five concentrations of icaritin for 24, 48 and $72 \mathrm{~h}$, respectively. Proteins were extracted in lysis buffer. Following quantification by ultraviolet spectrophotometry, $\sim 30-60 \mu \mathrm{g}$ total proteins in each lane were separated by SDS-PAGE (10 and $12 \%$ gels), and electrophoretically transferred onto nitrocellulose membranes. The membranes were blocked for $1 \mathrm{~h}$ in 5\% non-fat milk in TBS/T and then incubated with various autophagy antibodies in $3 \%$ non-fat milk at $4{ }^{\circ} \mathrm{C}$ overnight (1:500; Cell Signaling Technology). The next day, the membranes were washed three times for $10 \mathrm{~min}$, incubated in secondary antibody of anti-rabbit $\operatorname{HRP}(1: 10,000)$, washed again three times for $10 \mathrm{~min}$, and finally exposed to enhanced chemiluminescence (ECL) detection reagents (Amersham Pharmacia Biotech), and photographed.

Synergistic effect. The synergistic effect of drug combinations was evaluated by Chou-Talalay combination index (CI) equation as follows: $C I=D_{a} /\left(D_{x}\right)_{a}+D_{b} /\left(D_{x}\right)_{b}(27)$, where $D_{a},\left(D_{x}\right)_{a}$, $\mathrm{D}_{\mathrm{b}}$ and $\left(\mathrm{D}_{\mathrm{x}}\right)_{\mathrm{b}}$ indicate the $\mathrm{IC}_{50}$ value of drug $\mathrm{A}$ and $\mathrm{B}$ combination, drug $\mathrm{A}$ alone, $\operatorname{drug} \mathrm{B}$ and $\mathrm{A}$ combination and drug $\mathrm{B}$ alone, respectively. $\mathrm{CI}$ value of $<1,1$, or $>1$ indicate a synergic, additive, or antagonistic effect of two drugs, respectively.

Statistical analysis. The SPSS 19.0 (SPSS, Inc.) was used to carry out statistical analyses. The data are shown as mean \pm SD. Statistically significant differences between 
A<smiles>COc1ccc(-c2oc3c(CC=C(C)C)c(O)cc(O)c3c(=O)c2O)cc1</smiles>

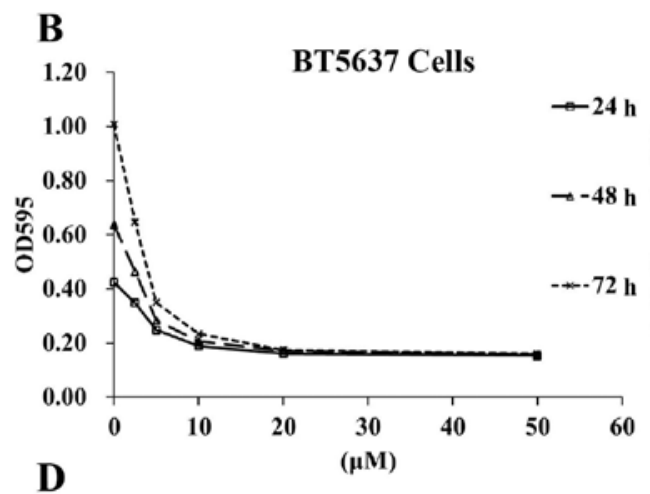

C

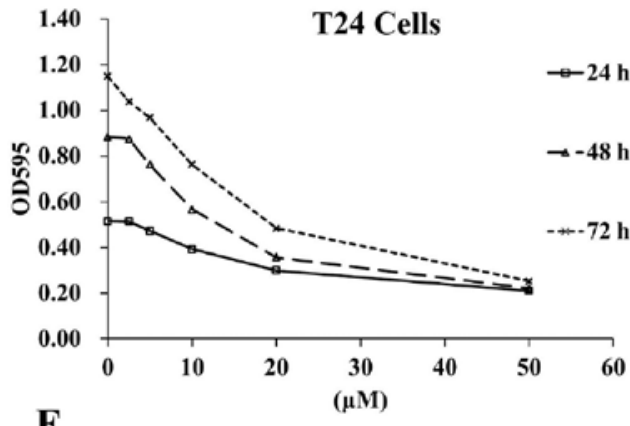

$\mathbf{E}$
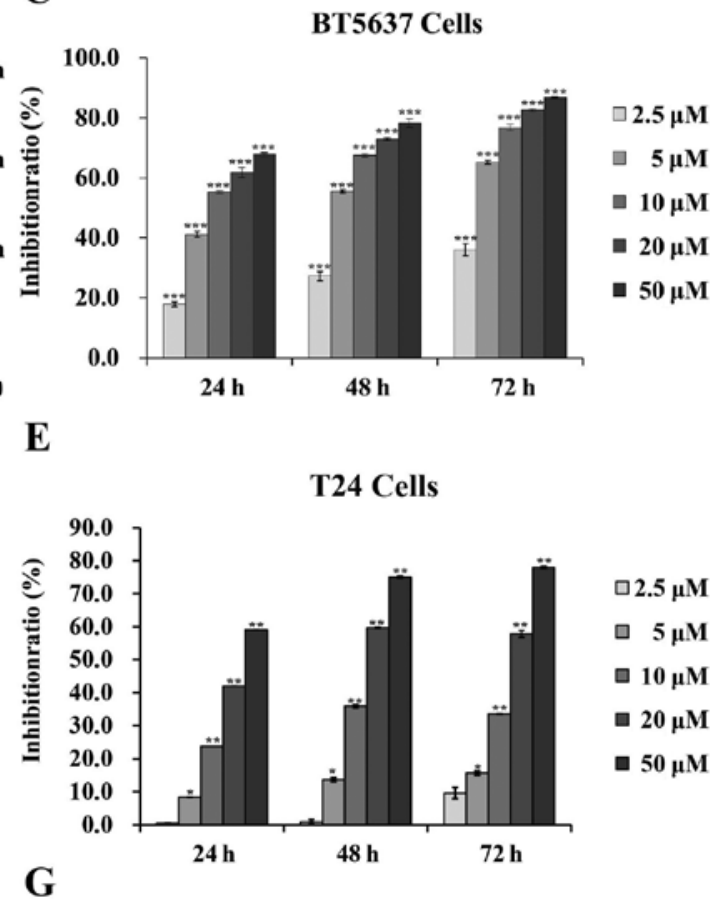

$\square 2.5 \mu \mathrm{M}$

$5 \mu \mathrm{M}$

- $10 \mu \mathrm{M}$

- $20 \mu \mathrm{M}$

- $50 \mu \mathrm{M}$

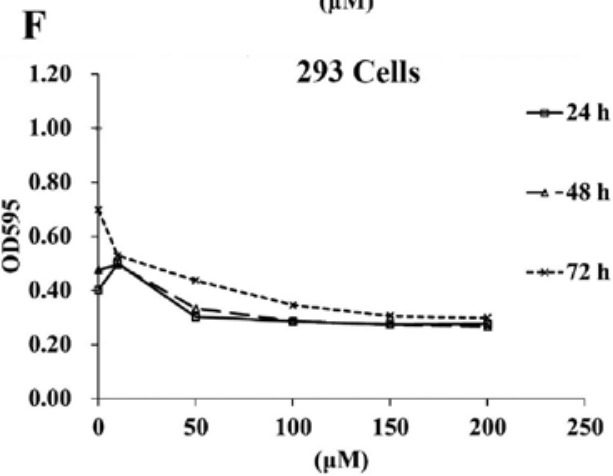

293 Cells

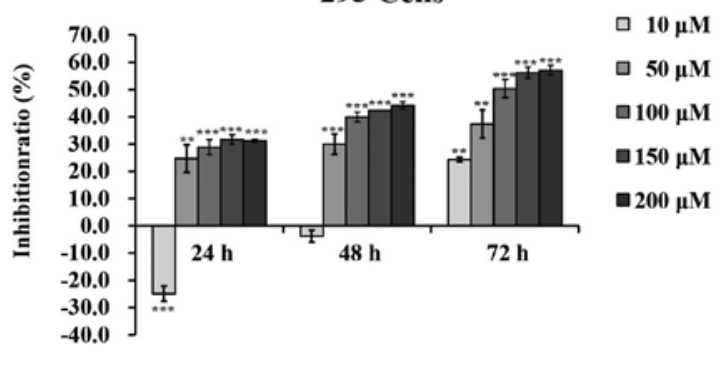

Figure 1. Icaritin time- and dose-dependently inhibits the proliferation of BT5637 and T24 cells. (A) The chemical structure of icaritin. (B-G) A total of

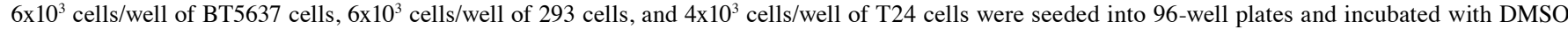
vehicle control or varying concentrations of icaritin for 24,48 and $72 \mathrm{~h}$. Proliferation of cells was evaluated using MTT assay. (B and C) Effects of icaritin on the proliferation of BT5637 cells. (D and F) Effects of icaritin on the proliferation of T24 cells. (E and G) Effects of icaritin on the proliferation of 293 cells. Data are expressed as mean $\pm \mathrm{SD},{ }^{*} \mathrm{P}<0.05,{ }^{* *} \mathrm{P}<0.01,{ }^{* * *} \mathrm{P}<0.001$ vs. DMSO vehicle control.

groups were analyzed using Student's t-test and $\mathrm{P}<0.05$ was considered to indicate a statistical significant result.

\section{Results}

Icaritin time- and dose-dependently inhibits proliferation of bladder cancer cells. To explore the effect of icaritin in bladder cancer cells, a dose-time-effect study was performed in BT5637 and T24 cells treated with concentration gradient of icaritin $(2.5,5,10,20$ and $50 \mu \mathrm{M})$ for 24,48 and $72 \mathrm{~h}$. As shown in Fig. 1B-F, icaritin inhibited the proliferation of both BT5637 and T24 cells dose- and time-dependently, as compared with DMSO vehicle control.

To verify the specificity of icaritin on bladder cancer cells rather than on normal human cells, we explored the $\mathrm{IC}_{50}$ of icaritin on normal human HEK293 cells. As shown in 
A

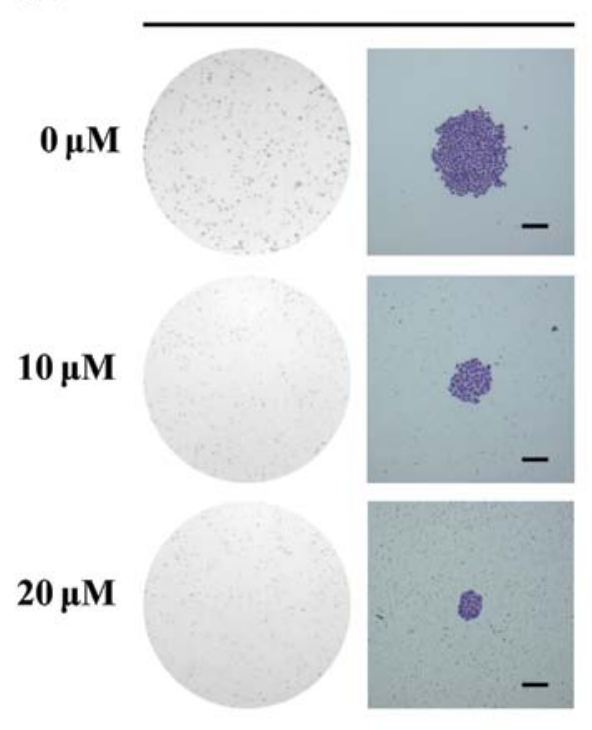

B

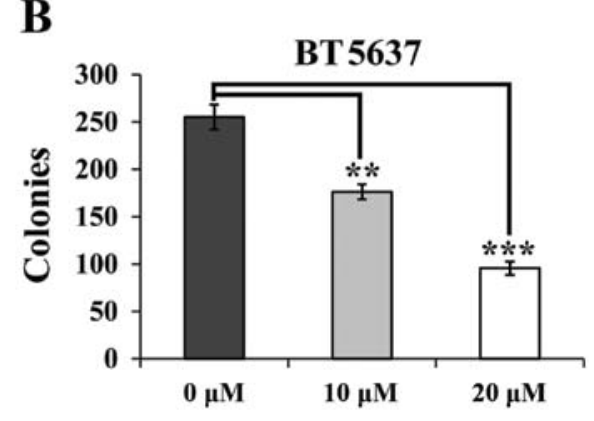

BT 5637
C

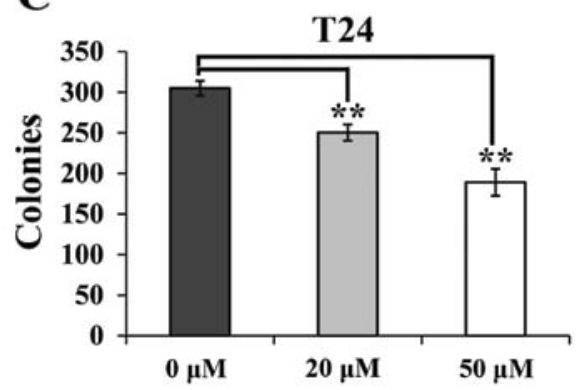

Figure 2. Icaritin inhibits the colony formation of BT5637 and T24 cells. BT5637 and T24 cells (6x10² cells/well) were seeded into 6-well plates and cultured with DMSO vehicle control or different concentrations of icaritin for $48 \mathrm{~h}$. (A) Colony-forming ability was evaluated by colony formation assay. (B and C) The size and number of colonies for BT5637 and T24 cells in different concentrations of icaritin were significantly lower than those in DMSO vehicle control $\left({ }^{*} \mathrm{P}<0.05,{ }^{* *} \mathrm{P}<0.01,{ }^{* * *} \mathrm{P}<0.001\right)$. Scale bar, $150 \mu \mathrm{m}$.

Fig. 1F and G, cell proliferation was minimally suppressed in 293 cells after icaritin treatment for 24 and $48 \mathrm{~h}$. The $\mathrm{IC}_{50}$ of icaritin on 293 cells at $72 \mathrm{~h}$ was $126.58 \mu \mathrm{M}$. Whereas, the $\mathrm{IC}_{50}$ of icaritin was 11.6, 7.21 and $4.46 \mu \mathrm{M}$ on BT5637 cells; 36.8, 21.7 and $20.3 \mu \mathrm{M}$ on T24 cells at 24,48 and $72 \mathrm{~h}$, respectively, indicating that cancer cells have a higher sensitivity towards icaritin than normal human cells, and that icaritin functioned as a promising anticancer agent with low cytotoxicity on normal cells.

Icaritin reduces the capacity of colony formation of bladder cancer cells. To confirm the effect of icaritin on colonyforming ability of bladder cancer cells, cell colony formation assay was performed on BT5637 and T24 cells treated with various concentrations of icaritin for $48 \mathrm{~h}$. As shown in Fig. 2A, the sizes of colony and the numbers of cells/colony in icaritin groups were smaller as compared with the DMSO vehicle control, particularly for BT5637 cells. Moreover, the result showed that the higher concentration of icaritin, the stronger the inhibitory effect. In addition, the number of colonies of BT5637 cells treated with icaritin at the concentrations of 10 and $20 \mu \mathrm{M}$ was $176.3 \pm 8.0$ and $95.7 \pm 7.1$, respectively, and that in T24 cells treated with icaritin at the concentrations of 20 and $50 \mu \mathrm{M}$ was $250.3 \pm 10$ and $189.0 \pm 16.5$, respectively, which was significantly decreased comparing to the DMSO vehicle groups in BT5637 and T24 cells $(255.3 \pm 13.0$ and $305 \pm 9.2$, respectively) (Fig. 2B). These results suggest that icartin suppressed the capacity of colony formation of bladder cancer cells.

Icaritin reduces mitochondrial membrane potential (MMP) in bladder cancer cells. Mitochondria, known as 'the power house', are important cellular structures that generate adenosine triphosphate (ATP) to supply most cellular functions. They are closely associated with cell cycle progression, cell growth and cell death (28). Therefore, we carried out JC-1 staining assay to evaluate whether icaritin could affect mitochondrial function in BT5637 and T24 cells. The result showed that MMP was reduced in BT5637 and T24 cells treated with icaritin (Fig. 3A). As shown in Fig. 3B and C, the cell viability in BT5637 cells were significantly decreased in 10 and $20 \mu \mathrm{M}$ icaritin groups as compared with the DMSO vehicle control $(94.88 \pm 0.06$ and $87.77 \pm 0.20$ vs. $98.35 \pm 0.10$, $\mathrm{P}<0.001$ ), and a similar result was seen in T24 cells treated with 20 and $50 \mu \mathrm{M}$ icaritin $(85.34 \pm 0.32$ and $76.4 \pm 10.18$ vs. $93.15 \pm 0.02, \mathrm{P}<0.001)$, indicating that the cell viability of bladder cancer cells was significantly inhibited after icaritin treatment in a dose-dependent manner. 
A

BT 5637

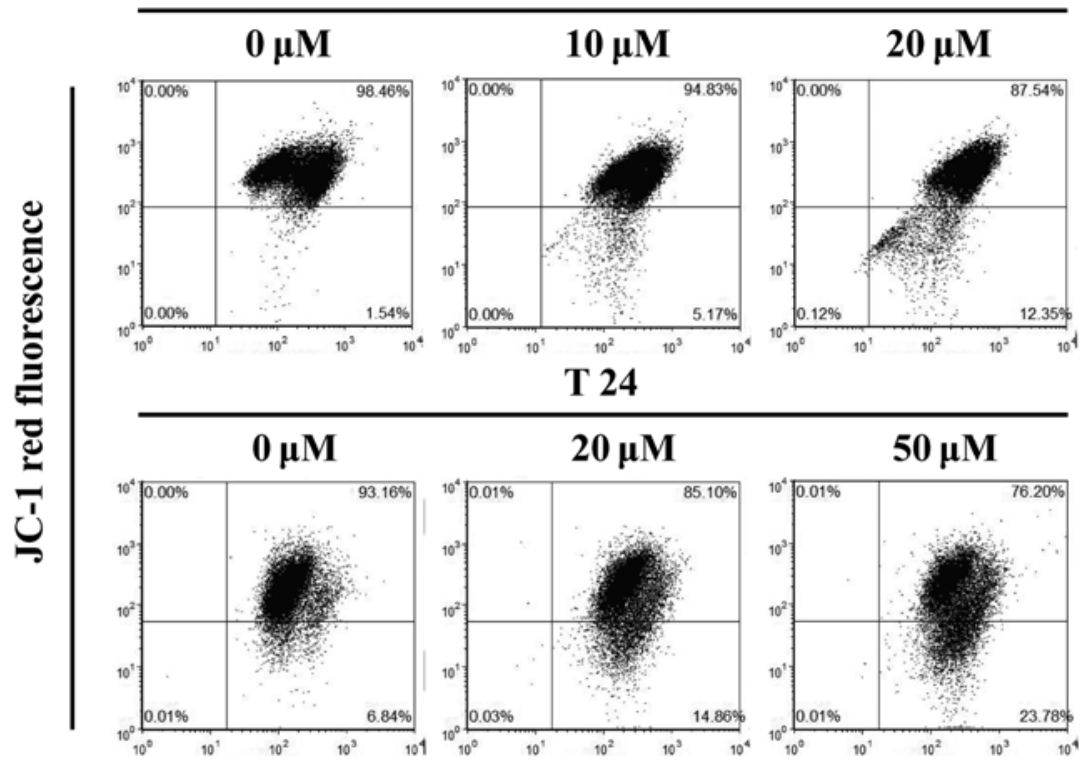

JC-1 green fluorescence

B

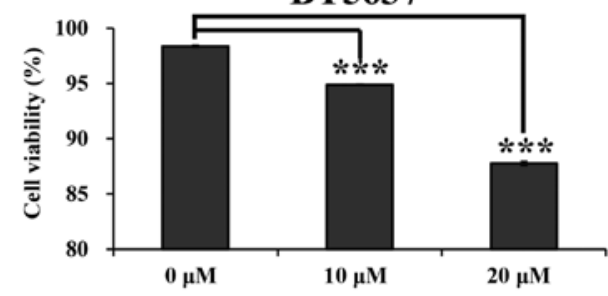

C

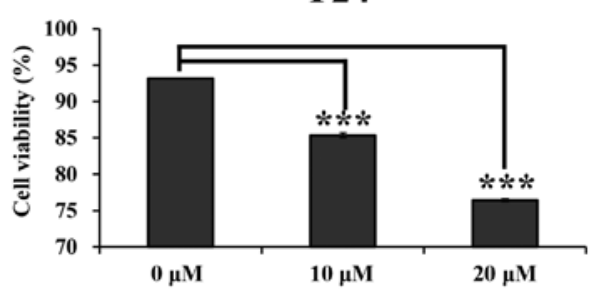

Figure 3. Icaritin decreases mitochondrial membrane potential (MMP) of BT5637 and T24 cells. BT5637 and T24 cells were incubated with DMSO vehicle control or varying concentrations of icaritin for $72 \mathrm{~h}$. The MMP of cells were evaluated by flow cytometry after labeling of JC-1 staining solution. (A) MMP was significantly decreased in BT5637 and T24 cells treated with icaritin compared with that in DMSO vehicle control. (B) The cell viability of bladder cancer cells in icaritin groups was significantly lower than the control group $\left.{ }^{* * *} \mathrm{P}<0.001\right)$.

Icaritin inhibits autophagy of bladder cancer cells. There was no significant change in cell cycle arrest and apoptosis of bladder cancer cells treated with icaritin (data not shown). To verify other potential mechanism of icaritin-induced cell inhibition, we examined the autophagy pathways and postulated that autophagy, which plays a vital role in cleaning injured mitochondria, may act as a major factor in regulating the growth of bladder cancer cells treated with icaritin. Using western blotting, major autophagy proteins including ATG3, ATG5, ATG7, ATG12 and LC3 were investigated to evaluate the autophagy levels of BT5637 and T24 treated with icaritin at 24, 48 and $72 \mathrm{~h}$. Given the lack of the duration of drug action (icaritin treatment for $24 \mathrm{~h}$ ), both bladder cancer T24 and BT5637 cells showed a slight difference in autophagy or even LC3 was initially upregulated in BT5637 (Fig. 4A). After 48-h treatment, the conversion of LC3-I to LC3-II was reduced (Fig. 4B), indicating that inhibition of autophagy in BT5637 and T24 cells treated with icaritin was initiated. Comparing with the DMSO vehicle control, autophagy of BT5637 and T24 cells significantly decreased in a dose-dependent manner, particularly at 48 and $72 \mathrm{~h}$ after 72 -h icaritin treatment. The above results suggest that icaritin inhibited the activation of constitutive autophagy.
Incaritin sensitizes bladder cancer cells to EPI by inhibiting EPI-induced autophagy. Knowing that inhibition of autophagy could sensitize cancer cells to chemotherapeutic agents $(21,22)$, we aimed to clarify whether combination of incaritin and EPI for treatment of BT5637 and T24 cells had a synergistic effect on cancer cells. To clarify this, we treated BT5637 and T24 bladder cancer cells with a combination of icaritin and EPI for $24 \mathrm{~h}$ and then detected the proliferation of these cells by MTT assay. The results in Fig. 5A and B showed that the $\mathrm{IC}_{50}$ value of icaritin alone $(7.07 \mu \mathrm{M})$ and EPI alone $(4.00 \mu \mathrm{g} / \mathrm{ml})$ in inhibiting BT5637 cells was significantly higher than that of icaritin in combination with $1.00 \mu \mathrm{g} / \mathrm{ml}$ EPI $(0.96 \mu \mathrm{M})$ and EPI in combination with $2.5 \mu \mathrm{M}$ icaritin $(1.57 \mu \mathrm{g} / \mathrm{ml})$. The same interesting results were also observed in T24 cells (Fig. 5C and D). To confirm whether combination of incaritin and EPI had a synergistic effect, we adopted the Chou-Talalay combination index (CI) equation. Based on the calculations, the CI value of icaritin and EPI combination in treating BT5637 and T24 cells was 0.53 and 0.56 , respectively (Fig. 5E and F), indicating that icaritin and EPI acted synergistically to suppress the proliferation of bladder cancer cells. To verify our result concerning the synergistic antitumor 


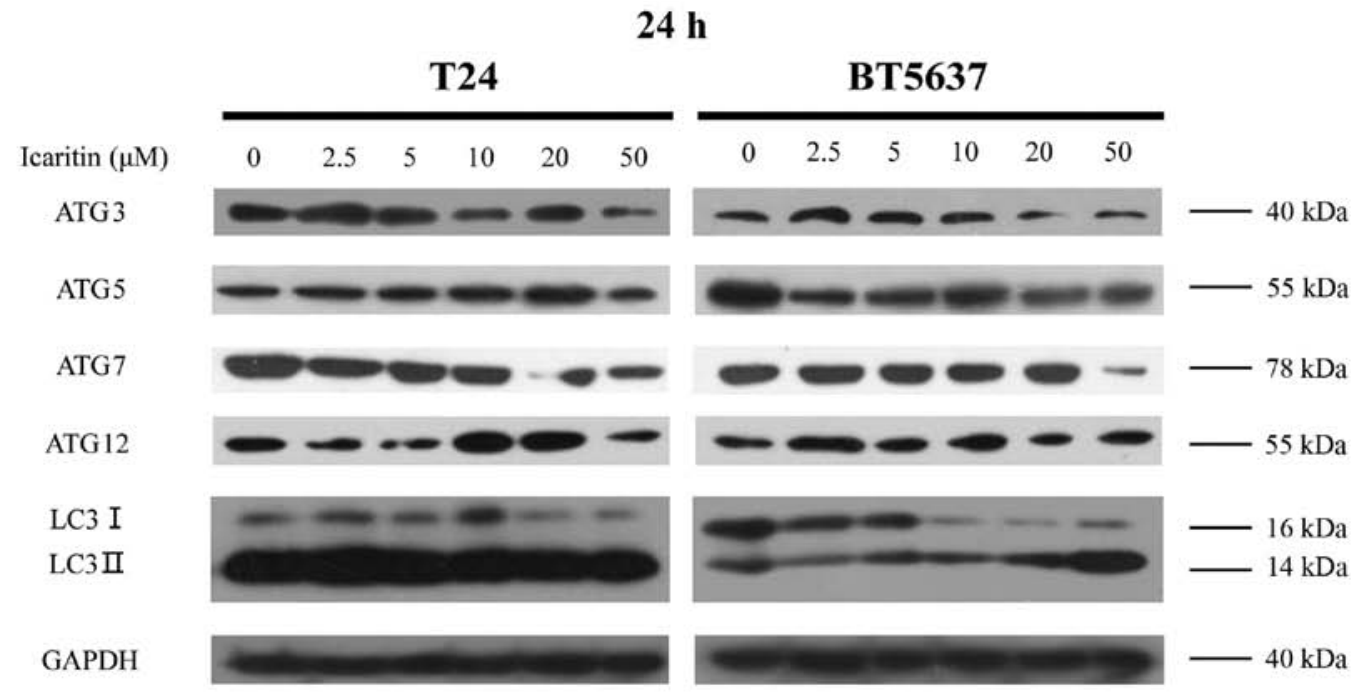

$48 \mathrm{~h}$

T24

Icaritin $(\mu \mathrm{M})$

\begin{tabular}{llllll}
\hline 0 & 2.5 & 5 & 10 & 20 & 50
\end{tabular}

\begin{tabular}{llllll}
\multicolumn{8}{c}{ BT5637 } \\
\hline \begin{tabular}{llllll}
\hline 0 & 2.5 & 5 & 10 & 20 & 50
\end{tabular}
\end{tabular}

ATG 3
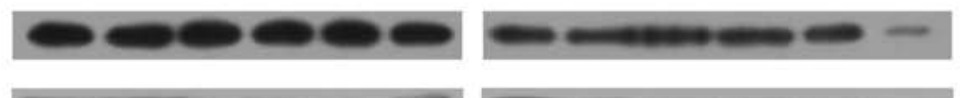

$-40 \mathrm{kD}$

ATG5

ATG 7
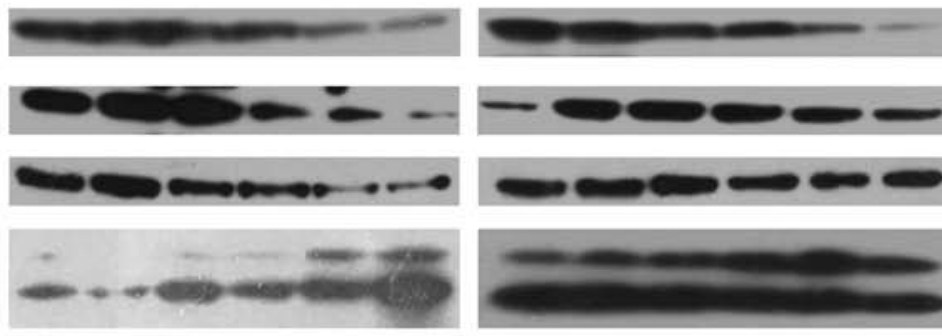

$-55 \mathrm{kD}$

ATG12

LC3 I

LC3 II

GAPDH
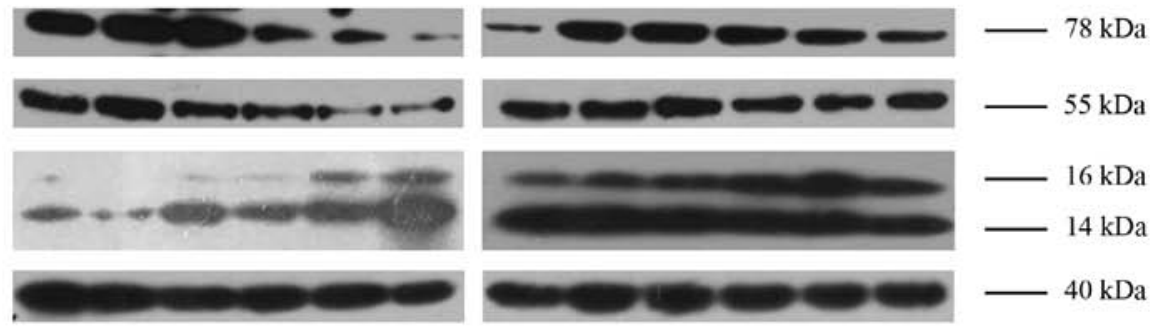

$72 \mathrm{~h}$

T24

\begin{tabular}{llllll}
\hline 0 & 2.5 & 5 & 10 & 20 & 50 \\
\hline & & & & &
\end{tabular}

BT5637

Icaritin $(\mu \mathrm{M})$

ATG 3

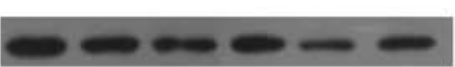

ATG 5

ATG 7

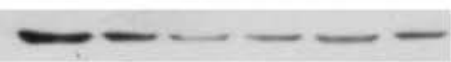

ATG12
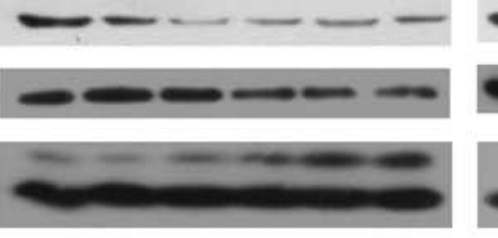

\begin{tabular}{llllll}
\multicolumn{6}{c}{ BT5637 } \\
\hline $\begin{array}{llllll}0 & 2.5 & 5 & 10 & 20 & 50\end{array}$
\end{tabular}

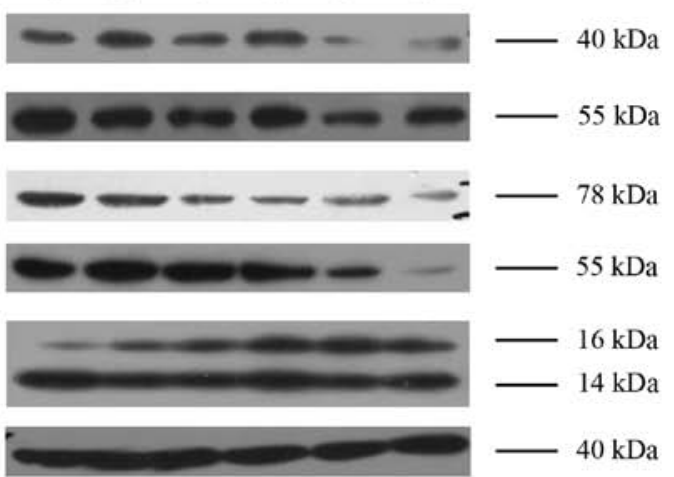

Figure 4. Icaritin induced autophagy suppression. Both bladder cancer cells T24 and BT5637 were treated with icaritin in 2.5, 5, 10,20 and 50 $\mu \mathrm{M}$ for 24,48 and $72 \mathrm{~h}$. Proteins of ATG8 conjugation system including ATG3, ATG5, ATG7 and ATG12 were detected. Additionally, both autophagosome marker LC3-I and LC3-II were discovered.

effect, western blotting was carried out to confirm whether incaritin inhibited EPI-induced autophagy and enhanced the susceptibility of bladder cancer cells to EPI. The result showed that icaritin not only suppressed the production of
LC3-I as an autophagic marker in cytoplasm but inhibited the conversion of LC3-I to LC3-II (Fig. 5E and F), suggesting that icaritin could sensitize bladder cancer cells to epirubicin treatment via downregulating EPI-induced autophagy. 
A

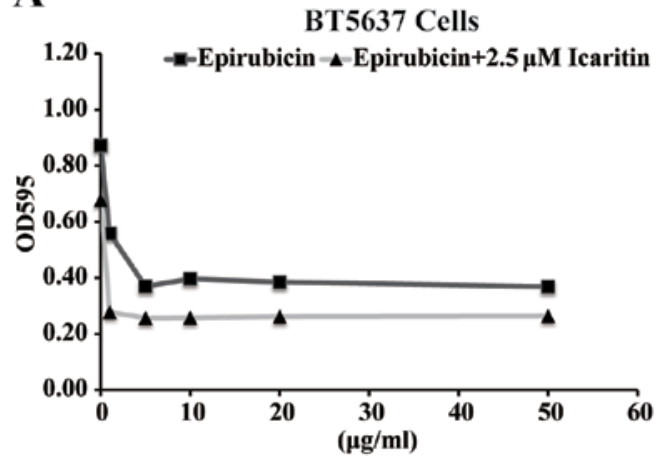

C

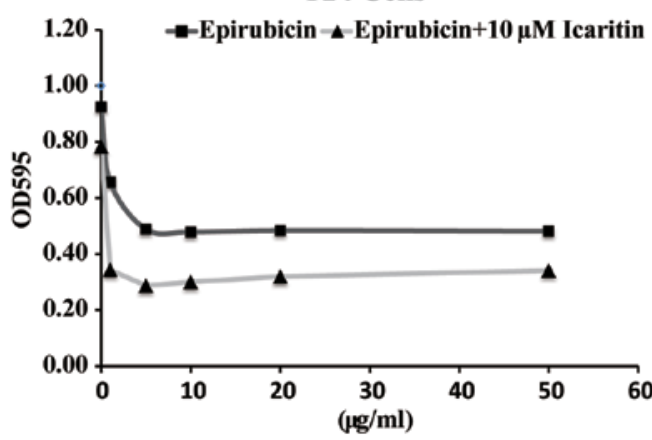

E

\begin{tabular}{ll}
\multicolumn{2}{c}{ BT5637 Cells } \\
\hline Drugs & IC 50 \\
\hline Icaritin & $7.07 \mu \mathrm{M}$ \\
Epirubicin & $4.00 \mu \mathrm{g} / \mathrm{ml}$ \\
Icaritin $+1 \mu \mathrm{g} / \mathrm{ml}$ Epirubicin & $0.96 \mu \mathrm{M}$ \\
Epirubicin $+2.5 \mu \mathrm{M}$ Icaritin & $1.57 \mu \mathrm{g} / \mathrm{ml}$ \\
\hline
\end{tabular}

Combination index $=0.53$

G

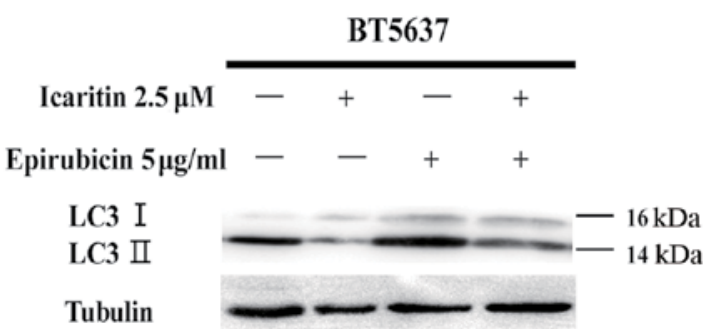

B

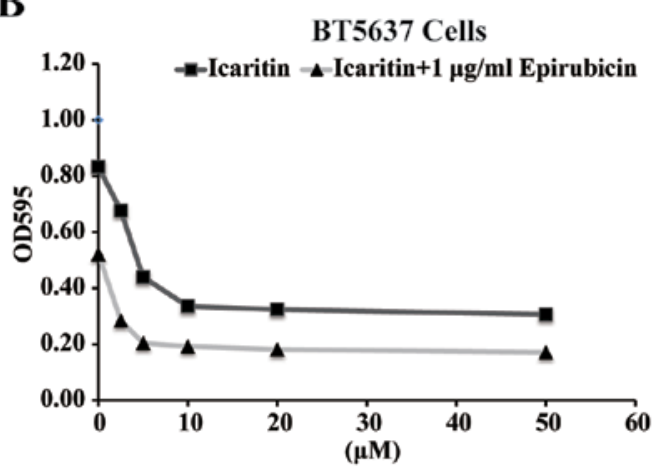

D

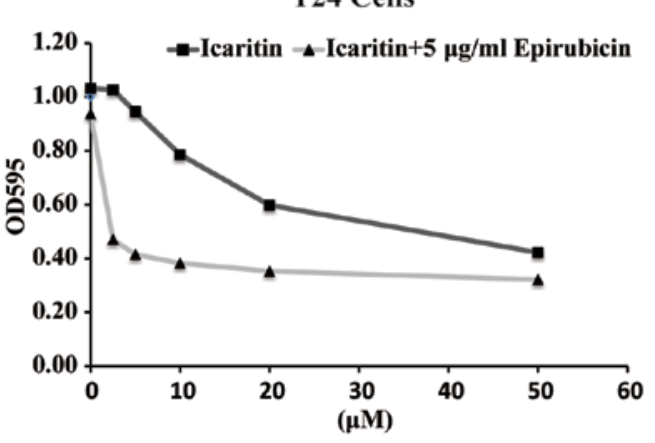

F

\begin{tabular}{lc}
\multicolumn{2}{c}{ T24 Cells } \\
\hline Drugs & IC 50 \\
\hline Icaritin & $36.81 \mu \mathrm{M}$ \\
Epirubicin & $9.92 \mu \mathrm{g} / \mathrm{ml}$ \\
Icaritin $+5 \mu \mathrm{g} / \mathrm{ml}$ Epirubicin & $3.09 \mu \mathrm{M}$ \\
Epirubicin $+10 \mu \mathrm{M}$ Icaritin & $4.71 \mu \mathrm{g} / \mathrm{ml}$ \\
\hline
\end{tabular}

Combination index $=\mathbf{0 . 5 6}$

H

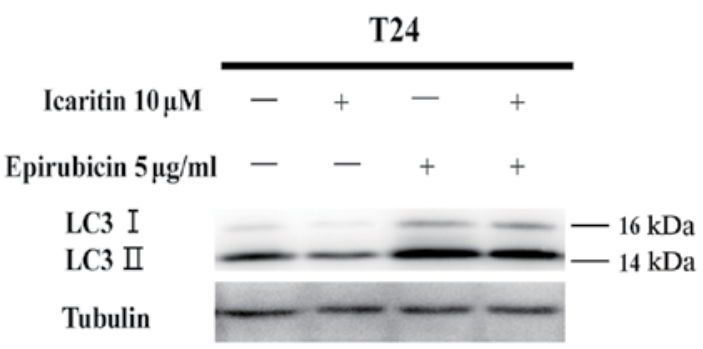

Figure 5. Icaritin synergistically potentiates the sensitivity of bladder cancer BT5637 and T24 cells to EPI. A total of 1x104 cells/well BT5637 cells, and $8 \times 10^{3}$ cells/well T24 cells were seeded into 96-well plates and cultured with icaritin alone, icaritin + EPI (BT5637 cells, $1 \mu \mathrm{g} / \mathrm{ml} ;$ T24 cells, $\left.5 \mu \mathrm{g} / \mathrm{ml}\right)$, EPI alone and EPI + icaritin (BT5637 cells, $2.5 \mu \mathrm{M}$; T24 cells, $10 \mu \mathrm{M}$ ) for $24 \mathrm{~h}$. The effects of combination were evaluated by MTT assay. The proliferation of BT5637 and T24 cells undergoing combination therapy was significantly decreased compared with those undergoing either icaritin or EPI treatment alone (A-D). The CI value of BT5637 and T24 cells treated with icaritin + EPI was $<1$ (E and F), showing a synergic effect. Further investigation on the protein level of autophagy showed that icaritin played a vital role in combination therapy with EPI ( $\mathrm{G}$ and $\mathrm{H})$.

\section{Discussion}

Bladder cancer is notorious for its high recurrence rate. Although a routine intravesical perfusion with EPI after surgery may reduce the recurrence rate, the acquired resistance to EPI is common in cancer patients (29). Therefore, increased attention has been paid to uncover the mechanism of drug resistance, and seeking novel agents among natural herbal ingredients (29).
In the present study, in an effort to find effective compounds to suppress the malignant proliferation of bladder cancer cells, we screen a library of small compound extracted from herbal medicine. We identified that icaritin may inhibit the proliferation of T24 and BT5637 cells. Furthermore, as it has been reported that icariin, an analogue of icaritin, may induce apoptosis in human hepatoma SMMC-7721 cells via a ROS/JNK-dependent mitochondrial pathway (30), we also investigated the effect of icariin on bladder cancer cells. 
However, differently to that previously reported in hepatoma cells, icariin showed a very low cytotoxicity on bladder cancer cells compared to icaritin. Therefore, icaritin was chosen to further explore the antitumor molecular mechanism in bladder cancer cells. Icaritin significantly inhibited the proliferation and colony formation of bladder cancer cells in a time- and dose-dependent manner. BT5637 cells ( $\mathrm{IC}_{50}$ at 24, 48 and $72 \mathrm{~h}, 11.6,7.21$ and $4.46 \mu \mathrm{M})$ showed higher sensitivity to icaritin than $\mathrm{T} 24$ cells $\left(\mathrm{IC}_{50}\right.$ at 24,48 and $72 \mathrm{~h}, 36.8,21.7$ and $20.3 \mu \mathrm{M}$ ).

In order to explore the molecular mechanism how icaritin inhibited the proliferation of BT5637 and T24 cells, we analyzed the cell cycle distribution and apoptosis of both cells using PI/Annexin V and flow cytometric analysis. However, the results showed that icaritin hardly affected cell cycle distribution or apoptosis in the cells (data not shown). However, we discovered that mitochondrial membrane potential (MMP) of BT5637 and T24 cells was obviously impaired by icaritin treatment. Mitochondria are known to play a vital role in cell growth as they provide large amounts of ATP for various cellular activities (27). Since tumor cells show extremely active growth, large amounts of ATP are required for tumor cells to support malignant cell proliferation (31). Therefore, decrease of mitochondrial membrane potential may lead to a reduced ATP production and suppressed cell proliferation rate. In the present study, growth inhibitory effect of icaritin in bladder cancer cells may be mediated through MMP suppression.

For eukaryotic cells, autophagy is a crucial pathway to recycle organelles and proteins, especially injured mitochondria. An elevated autophagic activity is utilized by tumor cells to acquire malignant proliferation. The accumulation of injured mitochondria in icaritin-treated bladder cancer cells prompted us to analyze autophagy in these cells. During autophagy, microtubule-associated protein 1A/1B-light chain 3 (LC3) is conjugated to phosphatidylethanolamine to form LC3-phosphatidylethanolamine (LC3-II)-conjugated by two consecutive ubiquitylation-like reactions catalyzed by the E1-like enzyme Atg7 and the E2-like enzyme Atg3 (32,33). LC3-II is recruited to autophagosomal membranes and degraded in autolysosomes after being fused to lysosomes $(32,33)$. Therefore, the protein levels of ATG3, ATG7, LC3 and LC3-II have been demonstrated to be reliable markers to monitor autophagy $(32,33)$. Moreover, ATG12, as the first ubiquitin-like ATG protein to be found, could bind to and was activated by ATG7, an E1-like enzyme (34). The ATG12 is sent to ATG10, an E2 enzyme, and finally forms a conjugation with ATG5, and then the ATG12-ATG5 conjugation futher coacts with ATG16, a small coiled-coil protein, and forms the ATG12-ATG5-ATG6 multimeric complex (34). We found that icaritin downregulated the expressions of ATG3, ATG7, ATG5, ATG12 and suppressed the transformation of LC3 to LC3-II, indicating that icaritin inhibited autophagy of bladder cancer cells.

Previous studies reported that ERK and AKT signaling are the dominant pathway for icaritin to inhibit cancer cells growth in chronic myeloid leukemia, endometrial cancer renal cell carcinoma and breast cancer cells $(15,17,18,35)$. Moreover, Li et al revealed that the apoptosis of human hepatoma was also induced via icariin treatment with ROS/JNK-dependent mitochondrial pathway (30). The above signaling pathways of icaritin were explored in the present study, however, the inhibition of bladder cancer cells undergoing icaritin treatment had no correlation with the signaling (data was not shown). We revealed that icaritin inhibited the autophagy pathway to effective inhibition of bladder cancer cells.

Autophagy is activated in tumor cells compared to normal cells, in response to cellular stress and/or increased metabolic demands, leading to rapid cell proliferation and acquired resistance to chemotherapies (19). Therefore, autophagy which is differentially regulated in cancer and normal cells may serve as a promising target for anticancer therapy. We found that icaritin significantly inhibited the proliferation of bladder cancer cells via downregulated autophagy. However, the $\mathrm{IC}_{50}$ value of icaritin for normal human HEK293 cells at $72 \mathrm{~h}$ was 28.4- and 6.2-fold higher than that for BT5637 and T24 cells at $72 \mathrm{~h}$, respectively, suggesting that human bladder cancer BT5637 and T24 cells which have active autophagic activity were more sensitive to icaritin compared to normal human HEK293 cells, and that icaritin may serve as an anticancer agent with low adverse effects.

In clinical practice, intravesical perfusion with epirubicin is routinely used after transurethral resection to prevent bladder cancer recurrence. Nevertheless, there exists an even more serious problem regarding EPI-resistance which limits its successful anticancer effect. In addition, adverse events accompanying high-dose intravesical usage of epirubicin, including chemical cystitis, urinary tract infection, hematuria and cardiac adverse events, have also been shown as a problem for bladder cancer patients (6). Therefore, a combined usage of an agent that can improve the sensitivity of EPI may reduce the side-effects due to high-dose EPI treatment. In the present study, we found that when combined with icaritin, EPI reached the same inhibitory effect on bladder cancer cells at a much lower dose compared to EPI treatment alone, suggesting that icaritin may serve as an adjuvant agent to boost EPI therapy. Recently, Chittaranjan et al found a similar result that EPI-resistant breast cancers cells treated with combination of EPI and autophagy inhibitor (hydroxychloroquine) markedly enhanced the inhibition of tumor growth comparing to those treated with EPI alone (36).

Autophagy is a major way that tumor cells utilize to develop chemoresistances to EPI. It is reported that EPI induces autophagy in human breast cancer MCF-7 cells, and EPI-induced autophagy protects MCF-7 cells from EPI-induced apoptosis (20). Also, EPI induces autophagy and serves as a pro-survival mechanism in triple negative breast cancers (TNBCs) (36). However, it has not been reported whether EPI may cause autophagy in bladder cancer. In the present study, we found that EPI treatment promoted the conversion of LC3I to LC3II, a marker of autophagosome (Fig. 5G and H), implying that EPI may induce autophagy in bladder cancer cells. As identified in Fig. 4 that icaritin acted as an autophagy inhibitor, we tested whether icaritin can suppress autophagy to increase EPI sensitivity in bladder cancer cells. The results showed that EPI-inducing autophagy was suppressed by icaritin treatment. Therefore, we found a novel compound icaritin functioning as an autophagy inhibitor to restore the sensitivity of bladder cancer to EPI.

In conclusion, the present study proposes that icaritin inhibited the malignant proliferation of bladder cancer cells 
through inhibition of autophagy. Moreover, as an autophagy inhibitor, icaritin functioned synergistically with EPI to treat bladder cancer cells by depressing EPI-induced autophagy. These results provide reasonable evidence that the natural product icaritin may prove to be a novel potent anticancer agent against bladder cancer.

\section{Acknowledgements}

The present study was supported by grants from the National Natural Science Foundation of China for Youths (no. 81202020), the National Natural Science Foundation of China (no. 81170637), the Shanghai Committee of Science and Technology General Program for Medicine (no. 11JC1402302), the Military Fund for Health Care (13BJZ29), and the Key Project of Science and Innovation Foundation of Shanghai Ministry of Education (14zz084).

\section{References}

1. Siegel R, Ma J, Zou Z and Jemal A: Cancer statistics, 2014. CA Cancer J Clin 64: 9-29, 2014.

2. Kaufman DS, Shipley WU and Feldman AS: Bladder cancer. Lancet 374: 239-249, 2009.

3. Parekh DJ, Bochner BH and Dalbagni G: Superficial and muscleinvasive bladder cancer: Principles of management for outcomes assessments. J Clin Oncol 24: 5519-5527, 2006.

4. Herr HW: Natural history of superficial bladder tumors: 10- to 20-year follow-up of treated patients. World J Urol 15: 84-88, 1997.

5. Rübben H, Lutzeyer W, Fischer N, Deutz F, Lagrange W and Giani G: Natural history and treatment of low and high risk superficial bladder tumors. J Urol 139: 283-285, 1988.

6. Onrust SV, Wiseman LR and Goa KL: Epirubicin: A review of its intravesical use in superficial bladder cancer. Drugs Aging 15 307-333, 1999

7. Wu H, Lien EJ and Lien LL: Chemical and pharmacological investigations of Epimedium species: A survey. Prog Drug Res 60: 1-57, 2003.

8. Wang Z, Zhang X, Wang H, Qi L and Lou Y: Neuroprotective effects of icaritin against beta amyloid-induced neurotoxicity in primary cultured rat neuronal cells via estrogen-dependent pathway. Neuroscience 145: 911-922, 2007.

9. Wang Z, Wang H, Wu J, Zhu D, Zhang X, Ou L, Yu Y and Lou Y: Enhanced co-expression of beta-tubulin III and choline acetyltransferase in neurons from mouse embryonic stem cells promoted by icaritin in an estrogen receptor-independent manner. Chem Biol Interact 179: 375-385, 2009.

10. Wo YB, Zhu DY, Hu Y, Wang ZQ, Liu J and Lou YJ: Reactive oxygen species involved in prenylflavonoids, icariin and icaritin initiating cardiac differentiation of mouse embryonic stem cells. J Cell Biochem 103: 1536-1550, 2008.

11. Zhu DY and Lou YJ: Inducible effects of icariin, icaritin, and desmethylicaritin on directional differentiation of embryonic stem cells into cardiomyocytes in vitro. Acta Pharmacol Sin 26: 477-485, 2005.

12. Huang J, Yuan L, Wang X, Zhang TL and Wang K: Icaritin and its glycosides enhance osteoblastic, but suppress osteoclastic, differentiation and activity in vitro. Life Sci 81: 832-840, 2007.

13. Chen MF, Qi L, Li Y, Zu XB, Dai YQ and Zhang P: Icaritin induces growth inhibition and apoptosis of human prostatic smooth muscle cells in an estrogen receptor-independent manner. Amino Acids 38: 1505-1513, 2010.

14. Hong J, Zhang Z, Lv W, Zhang M, Chen C, Yang S, Li S, Zhang L, Han D and Zhang W: Icaritin synergistically enhances the radiosensitivity of 4T1 breast cancer cells. PLoS One 8: e71347, 2013.

15. Tong JS, Zhang QH, Huang X, Fu XQ, Qi ST, Wang YP, Hou Y, Sheng J and Sun QY: Icaritin causes sustained ERK1/2 activation and induces apoptosis in human endometrial cancer cells. PLoS One 6: e16781, 2011

16. Huang X, Zhu D and Lou Y: A novel anticancer agent, icaritin, induced cell growth inhibition, G1 arrest and mitochondrial transmembrane potential drop in human prostate carcinoma PC-3 cells. Eur J Pharmacol 564: 26-36, 2007.
17. Guo $\mathrm{Y}$, Zhang $\mathrm{X}$, Meng $\mathrm{J}$ and Wang ZY: An anticancer agent icaritin induces sustained activation of the extracellular signalregulated kinase (ERK) pathway and inhibits growth of breast cancer cells. Eur J Pharmacol 658: 114-122, 2011.

18. Li S, Priceman SJ, Xin H, Zhang W, Deng J, Liu Y, Huang J, Zhu W, Chen M, Hu W, et al: Icaritin inhibits JAK/STAT3 signaling and growth of renal cell carcinoma. PLoS One 8: e81657, 2013.

19. Yang ZJ, Chee CE, Huang S and Sinicrope FA: The role of autophagy in cancer: Therapeutic implications. Mol Cancer Ther 10: 1533-1541, 2011.

20. Sun WL, Chen J, Wang YP and Zheng H: Autophagy protects breast cancer cells from epirubicin-induced apoptosis and facilitates epirubicin-resistance development. Autophagy 7: 1035-1044, 2011.

21. Xie JM, Li B, Yu HP, Gao QG, Li W, Wu HR and Qin ZH: TIGAR has a dual role in cancer cell survival through regulating apoptosis and autophagy. Cancer Res 74: 5127-5138, 2014.

22. Chen WC, Hsu KY, Hung CM, Lin YC, Yang NS, Ho CT, Kuo SC and Way TD: The anti-tumor efficiency of pterostilbene is promoted with a combined treatment of Fas signaling or autophagy inhibitors in triple negative breast cancer cells. Food Funct 5: 1856-1865, 2014

23. Tang Q, Li G, Wei X, Zhang J, Chiu JF, Hasenmayer D, Zhang D and Zhang $\mathrm{H}$ : Resveratrol-induced apoptosis is enhanced by inhibition of autophagy in esophageal squamous cell carcinoma. Cancer Lett 336: 325-337, 2013.

24. Cui Q, Tashiro S, Onodera S, Minami $M$ and Ikejima T: Autophagy preceded apoptosis in oridonin-treated human breast cancer MCF-7 cells. Biol Pharm Bull 30: 859-864, 2007.

25. Zhang XQ, Dunner K Jr and Benedict WF: Autophagy is induced by adenoviral-mediated interferon alpha treatment in interferon resistant bladder cancer and normal urothelial cells as a cell death protective mechanism but not by the bystander factors produced. Cancer Gene Ther 17: 579-584, 2010.

26. Sang H, Zhang L and Li J: Anti-benzopyrene-7,8-diol-9,10epoxide induces apoptosis via mitochondrial pathway in human bronchiolar epithelium cells independent of the mitochondria permeability transition pore. Food Chem Toxicol 50: 2417-2423, 2012.

27. Chou TC, Motzer RJ, Tong Y and Bosl GJ: Computerized quantitation of synergism and antagonism of taxol, topotecan, and cisplatin against human teratocarcinoma cell growth: A rational approach to clinical protocol design. J Natl Cancer Inst 86: 1517-1524, 1994.

28. McBride HM, Neuspiel M and Wasiak S: Mitochondria: More than just a powerhouse. Curr Biol 16: R551-R560, 2006.

29. Yang CC, Chen GW, Lu HF, Wang DY, Chen YS and Chung JG: Paclitaxel (taxol) inhibits the arylamine $\mathrm{N}$-acetyltransferase activity and gene expression (mRNA NAT1) and 2-aminofluorene-DNA adduct formation in human bladder carcinoma cells (T24 and TSGH 8301). Pharmacol Toxicol 92: 287-294, 2003

30. Li S, Dong P, Wang J, Zhang J, Gu J, Wu X, Wu W, Fei X, Zhang Z, Wang Y, et al: Icariin, a natural flavonol glycoside, induces apoptosis in human hepatoma SMMC-7721 cells via a ROS/JNK-dependent mitochondrial pathway. Cancer Lett 298: 222-230, 2010

31. Weinberg F and Chandel NS: Mitochondrial metabolism and cancer. Ann NY Acad Sci 1177: 66-73, 2009.

32. Mizushima N and Yoshimori T: How to interpret LC3 immunoblotting. Autophagy 3: 542-545, 2007.

33. Tanida I, Ueno T and Kominami E: LC3 and Autophagy. Methods Mol Biol 445: 77-88, 2008.

34. Yamaguchi M, Noda NN, Yamamoto H, Shima T, Kumeta H, Kobashigawa Y, Akada R, Ohsumi Y and Inagaki F: Structural insights into Atg10-mediated formation of the autophagy-essential Atg12-Atg5 conjugate. Structure 20: 1244-1254, 2012.

35. Zhu J, Li Z, Zhang G, Meng K, Kuang W, Li J, Zhou X, Li R, Peng H, Dai C, et al: Icaritin shows potent anti-leukemia activity on chronic myeloid leukemia in vitro and in vivo by regulating MAPK/ERK/JNK and JAK2/STAT3/AKT signalings. PLoS One 6: e23720, 2011.

36. Chittaranjan S, Bortnik S, Dragowska WH, Xu J, Abeysundara N, Leung A, Go NE, DeVorkin L, Weppler SA, Gelmon K, et al: Autophagy inhibition augments the anticancer effects of epirubicin treatment in anthracycline-sensitive and -resistant triple-negative breast cancer. Clin Cancer Res 20: 3159-3173, 2014. 